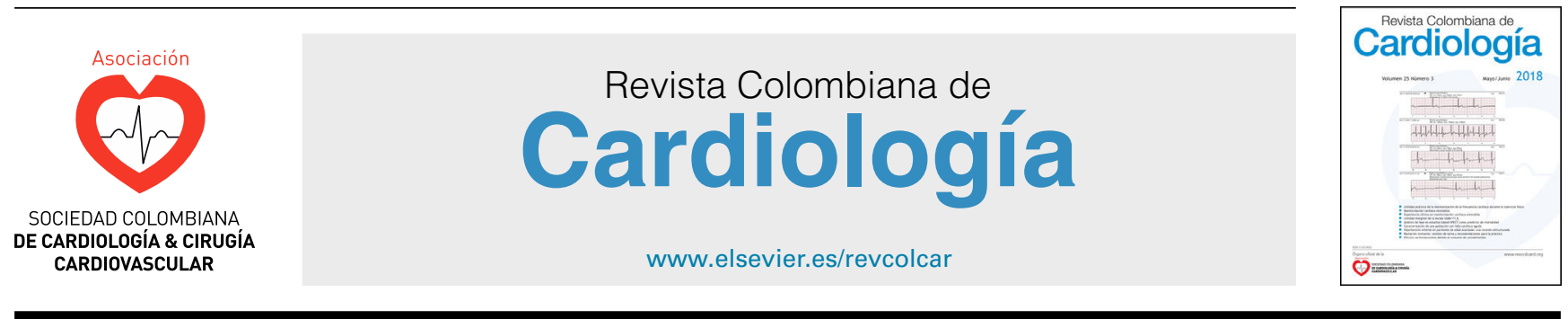

CARDIOLOGÍA DEL ADULTO - PRESENTACIÓN DE CASOS

\title{
Perfusión de furosemida subcutánea como tratamiento paliativo en insuficiencia cardiaca refractaria
}

\author{
Susana del Prado Díaz ${ }^{a}$, Miguel Ángel Sancho Zamora ${ }^{\mathrm{b}, *}$, Sergio Collazoc ${ }^{\mathrm{c}}$, \\ Isabel Cañada Millas ${ }^{\mathrm{b}}$ y José Luis Zamorano ${ }^{\mathrm{a}}$
}

\author{
a Servicio de Cardiología. Hospital Universitario Ramón y Cajal, Madrid, España \\ b Unidad de Cuidados Paliativos. Hospital Universitario Ramón y Cajal, Madrid, España \\ c Equipo de Soporte de Atención Paliativa Domiciliaria. Dirección Asistencial Este, Madrid, España
}

Recibido el 1 de septiembre de 2017; aceptado el 23 de septiembre de 2019

Disponible en Internet el 28 de enero de 2020

\section{PALABRAS CLAVE \\ Cuidados paliativos; Insuficiencia \\ cardiaca; \\ Diuréticos; \\ Infusión subcutánea; \\ Furosemida}

\section{KEYWORDS}

Palliative care;

Heart failure;

Diuretics;

Subcutaneous

infusion;

Furosemide

\begin{abstract}
Resumen En la insuficiencia cardiaca terminal los síntomas predominantes habitualmente están relacionados con la congestión; sin embargo, la resistencia a diuréticos vía oral es frecuente y necesita tratamiento parenteral para paliar los síntomas. La administración intravenosa de furosemida requiere hospitalización o asistencia hospitalaria, lo que supone un deterioro significativo de la calidad de vida de pacientes en fases avanzadas de la enfermedad.

La administración subcutánea continua de furosemida mediante infusor elastomérico es una técnica eficaz con baja tasa de complicaciones graves que permite la administración de diurético parenteral en el ámbito domiciliario, y evita la necesidad de hospitalizar. A pesar de que esta modalidad de tratamiento no está aceptada en ficha técnica y requiere más estudios que precisen su indicación, puede suponer un recurso adecuado para pacientes seleccionados.

Se presenta el caso de una paciente con insuficiencia cardiaca avanzada, síntomas congestivos y ausencia de respuesta a tratamiento deplectivo vía oral, tratada con perfusión continua subcutánea domiciliaria de furosemida mediante infusor elastomérico de forma prolongada.

(C) 2019 Sociedad Colombiana de Cardiología y Cirugía Cardiovascular. Publicado por Elsevier España, S.L.U. Este es un artículo Open Access bajo la licencia CC BY-NC-ND (http:// creativecommons.org/licenses/by-nc-nd/4.0/).
\end{abstract}

\section{Subcutaneous furosemide infusion as palliative treatment in refractory end-stage} heart failure

\begin{abstract}
In end-stage heart failure the predominant symptoms are usually associated with congestion. However, the resistance to oral diuretics is common and requires parenteral treatment to alleviate the symptoms. The intravenous administration of furosemide requires hospital admission or hospital care. This leads to a significant decrease in the quality of life of patients in advance stages of the disease.
\end{abstract}

\footnotetext{
* Autor para correspondencia.

Correo electrónico: masancho.hrc@salud.madrid.org (M.Á. Sancho Zamora).
} 
The continuous subcutaneous infusion of furosemide using an elastomeric pump is an effective technique, with a low rate of serious complications that allows a parenteral diuretic to be administered in the home environment and thus avoiding the need for hospital admission. Despite this mode of treatment not being indicated in the drug data sheet, and requires more studies that specify its indication, it may be a suitable recourse for selected patients.

It is presented a case of a patient with advanced heart failure, congestive symptoms, and a lack of response to oral diuretics treatment, and then treated with a continuous and prolonged subcutaneous infusion of furosemide at home using an elastomeric infusion pump.

(c) 2019 Sociedad Colombiana de Cardiología y Cirugía Cardiovascular. Published by Elsevier España, S.L.U. This is an open access article under the CC BY-NC-ND license (http:// creativecommons.org/licenses/by-nc-nd/4.0/).

\section{Introducción}

La insuficiencia cardiaca es un síndrome clínico cuya prevalencia aumenta con la edad, superando el $8 \%$ en población mayor de 70 años $^{1}$. En fases avanzadas es frecuente la aparición de síntomas difíciles de manejar, generalmente relacionados con la congestión. La administración de diuréticos es la base del tratamiento; sin embargo, el deterioro habitual de la función renal y el edema intestinal pueden comprometer la eficiencia diurética a pesar del incremento de las dosis o la combinación de fármacos. Se sabe que el deterioro de la respuesta diurética está directamente relacionado con la tasa de reingresos y mortalidad ${ }^{2}$, y que la ausencia de respuesta a diuréticos vía oral hacen necesaria su administración intravenosa, lo que suele requerir el ingreso hospitalario.

Previamente se ha reportado la administración de furosemida por vía subcutánea como terapia alternativa al tratamiento diurético convencional en pacientes con mala respuesta a la vía oral con el objetivo de evitar ingresos hospitalarios ${ }^{3}$. Sin embargo, hasta el momento esta vía de administración no ha sido aprobada debido a la escasez de estudios farmacocinéticos y farmacodinámicos que determinen su absorción y bioequivalencias respecto a otras vías de administración ${ }^{4}$, y fundamentalmente, a la alcalinidad de su $\mathrm{pH}$ que puede producir irritación del punto de punción, de manera que su uso se realiza "off label"'.

A continuación se presenta el caso de una paciente con insuficiencia cardiaca estadío $D$, en quien se administra, como tratamiento paliativo, infusión continua de furosemida subcutánea mediante infusor elastomérico, de forma prolongada en el tiempo y con carácter ambulatorio.

\section{Caso}

Paciente de sexo femenino, de 86 años de edad, diagnosticada de cardiopatía reumática no intervenida en fase dilatada, remitida a la unidad de insuficiencia cardiaca avanzada por refractariedad a tratamiento médico convencional.

Debutó con semiología de insuficiencia cardiaca un año antes, momento en el que se diagnosticó afectación reumática polivalvular: insuficiencia mitral reumática severa, insuficiencia tricúspide severa y doble lesión aórtica leve. Entonces ya se encontraba en fibrilación auricular, con dilatación severa biauricular, presentaba disfunción ventricular izquierda moderada e hipertensión pulmonar moderada-severa (fig. 1). Previamente era independiente para actividades básicas e instrumentales y no existían datos de deterioro cognitivo. Sin embargo, dada la edad, el riesgo quirúrgico, y de acuerdo con la paciente que no deseaba intervención quirúrgica, se decidió manejo médico de los síntomas de insuficiencia cardiaca.

En su primera visita en la unidad de insuficiencia cardiaca avanzada se encontraba disneica con leves esfuerzos, presentaba tendencia a la hipotensión y signos evidentes de congestión: ingurgitación yugular, crepitantes en bases y edemas con fóvea hasta la raíz de las extremidades inferiores, sin ascitis. La radiografía de tórax mostraba clara cardiomegalia y derrame pleural bilateral (fig. 2, A y B). En el electrocardiograma se objetivaba fibrilación auricular con respuesta ventricular controlada y bloqueo de rama izquierda (fig. 2C). En la analítica destacaba deterioro agudo de la función renal sobre insuficiencia renal crónica (tasa de filtrado glomerular $30 \mathrm{ml} / \mathrm{min}$ ) y péptido natriurético tipo $B$ elevado $(2.500 \mathrm{pg} / \mathrm{ml})$.

A pesar del incremento de la dosis de diurético vía oral y combinación de diuréticos (diuréticos de asa a dosis alta e inhibidores de la aldosterona) persistía congestiva en las visitas consecutivas. Por este motivo fue incluida en un protocolo de administración de dosis intermitentes semanales de diurético intravenoso en el hospital de día. Inicialmente presentó buena respuesta, con depleción adecuada de volumen, ausencia de trastornos electrolíticos y deterioro de la función renal. Sin embargo, tras conseguir la euvolemia y finalizar la administración de diurético intravenoso con retorno a la vía oral presentó nuevamente clínica de congestión, por lo cual requirió ingreso hospitalario debido a insuficiencia cardiaca y fracaso renal agudo.

Dadas las características de la paciente, insuficiencia cardiaca secundaria a cardiopatía valvular no corregible en estadío $D$ y la resistencia a diuréticos orales se planteó, de forma conjunta entre los equipos de cuidados paliativos hospitalarios e insuficiencia cardiaca, tratamiento ambulatorio mediante perfusión subcutánea continua de furosemida como medida paliativa de control de síntomas. 


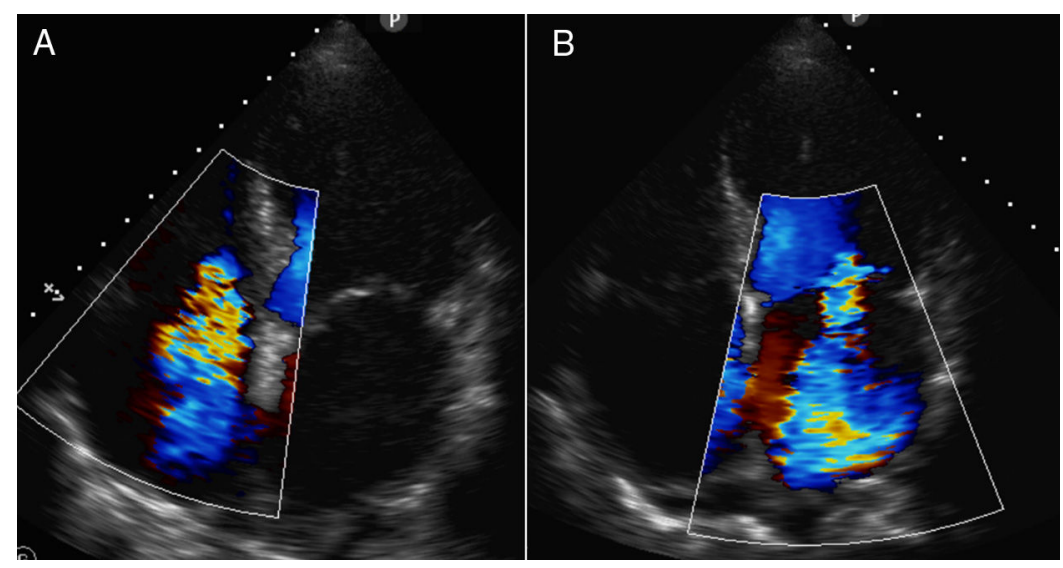

Figura 1 Ecocardiograma transtorácico en el que se objetiva afectación reumática polivalvular: insuficiencia tricúspide reumática severa $(A)$, insuficiencia mitral reumática severa $(B)$. Destaca la dilatación severa biauricular y la disfunción ventricular izquierda moderada.

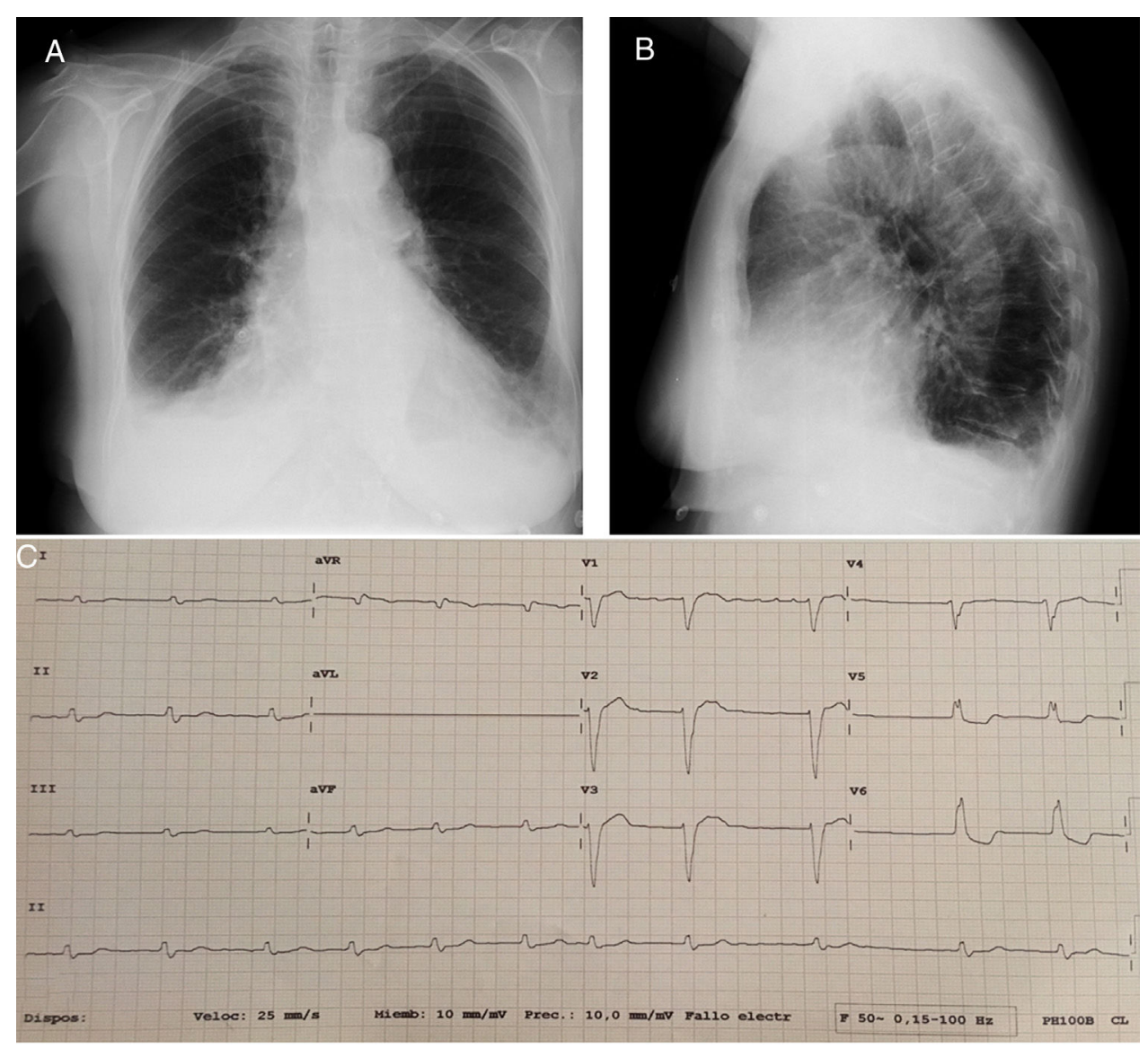

Figura 2 Radiografía de tórax posteroanterior (A) y lateral (B) que muestra cardiomegalia, derrame pleural bilateral y redistribución vascular compatible con insuficiencia cardiaca descompensada. (C). Electrocardiograma de 12 derivaciones que muestra fibrilación auricular con respuesta ventricular controlada y bloqueo de rama izquierda del haz de His.

Durante la hospitalización se empleó un infusor elastomérico modelo Accufuser ${ }^{\circledR}$ C0020L (Woo Young Medical Co., Ltd.) $300 \mathrm{ml}$ (fig. 3), que permite la infusión continua de fármacos a una velocidad constante y no modificable de $2 \mathrm{ml} / \mathrm{h}$, con duración aproximada de cinco días. Se cargó el infusor al $80 \%$ de su capacidad total $(240 \mathrm{ml})$, con una dilución de
$625 \mathrm{mg}$ de furosemida a concentración de $10 \mathrm{mg} / \mathrm{ml}$, completándose el volumen con $177,5 \mathrm{ml}$ de suero salino al $0,9 \%$ (dosis diaria de $125 \mathrm{mg}$ de furosemida subcutánea). La dosis fue ajustada en función de los requerimientos previos de furosemida intravenosa, evaluándose la respuesta diurética, la función renal y los iones previos al alta. 


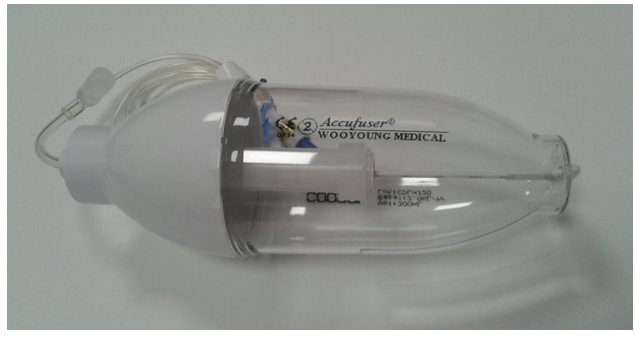

Figura 3 Infusor elastomérico modelo Accufuser $^{\circledR}$ CO020L (Woo Young Medical Co., Ltd.).

Se contactó con el equipo de cuidados paliativos domiciliarios para seguimiento y cambio de sistema elastomérico cada cinco días, con el objetivo de evitar la movilización frecuente de la paciente al hospital. Se planearon revisiones con analítica progresivamente espaciadas en el tiempo conforme a la estabilidad clínica. La dosis de furosemida se mantuvo idéntica en las revisiones sucesivas. El manejo de síntomas fue óptimo, con mejoría notable de la disnea y desaparición de edemas en miembros inferiores, así como ausencia de nuevas reagudizaciones y reingresos y mejoría de la función renal, con estabilidad electrolítica.

Durante las dieciséis semanas de tratamiento ininterrumpido, la única complicación presentada fue, en la semana 8 tras el inicio de la infusión continua, la aparición de enrojecimiento e induración no dolorosa de la zona de inserción del infusor subcutáneo, sugestiva de celulitis, que requirió cambio de la región de infusión a miembro superior contralateral y tratamiento antibiótico con amoxicilina/ácido clavulánico vía oral, con resolución completa.

La paciente sufrió una caída accidental con resultado de fractura humeral durante el tratamiento con furosemida subcutánea, aunque no se puede atribuir o descartar con seguridad que existiera relación entre ambas.

Finalmente, en visita domiciliaria, presentó deterioro del estado general y astenia, con incremento progresivo de malestar, por lo que se decidió retirar infusor subcutáneo y sedarla hasta su fallecimiento.

\section{Discusión}

La insuficiencia cardiaca o falla cardiaca terminal es un síndrome correspondiente al último estadío de diferentes entidades, caracterizado por alteraciones estructurales cardíacas y síntomas secundarios a la congestión venosa (pulmonar y/o sistémica) y al bajo gasto cardíaco a pesar de un correcto tratamiento dietético, restricción de sal y tratamiento médico máximo con la inclusión de IECA, ARA II, antagonistas de la aldosterona, betabloqueantes y diuréticos. La identificación de esta fase no siempre es fácil de realizar y su curso evolutivo es difícilmente predecible. Son frecuentes las visitas a los servicios de urgencias médicas y las hospitalizaciones repetidas que ocasionan pérdida de calidad de vida relacionada con la monitorización analítica, el tratamiento más o menos invasor y las normas de funcionamiento de los servicios de internamiento hospitalario. En fase terminal de la insuficiencia cardiaca, en la que las medidas modificadoras del pronóstico no son tan efectivas o no se consideran ya indicadas, los objetivos principales del tratamiento son: ofrecer soporte emocional al paciente $y$ familiares, planear la toma de decisiones, lograr la mayor calidad de vida posible y controlar los síntomas que producen malestar ${ }^{5,6}$. Entre los más frecuentes se destacan la disnea, los secundarios a congestión venosa sistémica (edemas, ascitis, anorexia), la astenia, la tristeza/ansiedad y el dolor.

Los diuréticos de asa son los fármacos más útiles para el tratamiento de la congestión venosa, tanto pulmonar como sistémica, pero en fases avanzadas en las que existe también edema intestinal no se produce una adecuada absorción y la biodisponibilidad cae llamativamente con la consiguiente pérdida de eficacia. En estos casos resulta necesaria la administración parenteral, bien en pulsos o en infusión continua, para mantener la respuesta diurética. Con el fin de minimizar en lo posible el menoscabo de la calidad de vida y las molestias relacionadas con la hospitalización se puede proponer el tratamiento sintomático de forma ambulatoria o, mejor aún, en el domicilio del paciente con la colaboración de los equipos de atención primaria, de cuidados paliativos $u$ otros equipos de hospitalización a domicilio ${ }^{7}$.

La dificultad del tratamiento con furosemida parenteral en el ámbito domiciliario estriba en que su uso intravenoso, ajustado a las indicaciones autorizadas en la ficha técnica del medicamento, resulta inviable en períodos prolongados, pues obligaría a una asistencia muy continuada mediante equipos de hospitalización a domicilio.

Esta dificultad se puede solventar con la administración "off label" de furosemida por vía subcutánea ${ }^{8}$ que parece demostrar efectividad para forzar la diuresis, producir depleción de volumen y así controlar los síntomas congestivos en pacientes con insuficiencia cardiaca avanzada y terminal.

La evidencia científica es escasa hasta el momento, reduciéndose a algunas comunicaciones de cohortes de pequeño tamaño $0^{3,9,10}$, y no existen indicaciones claras acerca de qué subgrupos de pacientes son más susceptibles de respuesta, cuál sería el régimen de dosificación eficaz y cuál la logística de la administración del fármaco ${ }^{11}$. Tampoco están nítidamente definidos los riesgos y beneficios de esta vía de administración, pero parece una opción válida para pacientes seleccionados en situación de insuficiencia cardiaca terminal que desean evitar el internamiento hospitalario y en quienes los objetivos se centran en el control sintomático. La furosemida utilizada fue la presentación convencional para uso intravenoso que tiene un $\mathrm{pH}$ alcalino, irritante para el tejido cutáneo. Recientemente se ha comunicado el desarrollo de una nueva formulación de $\mathrm{pH}$ más neutro ${ }^{12}$ que estaría específicamente indicada para uso subcutáneo y que podría limitar las reacciones adversas locales.

En el caso presentado, a la paciente, conocedora del diagnóstico y del mal pronóstico y con deseos manifiestos de evitar los ingresos en la medida de lo posible, se le ofreció la posibilidad de administración subcutánea de furosemida para controlar sus síntomas advirtiéndole de que se trataba de una modalidad terapéutica que, aunque aparentemente segura y efectiva, aún no se encuentra universalmente aceptada.

Con su consentimiento se inició el tratamiento en el hospital. Una vez calculada la dosis efectiva diaria de furosemida intravenosa según respuesta diurética y con control analítico de iones y función renal, se comenzó a administrar la misma dosis por vía subcutánea a través de un infusor 
elastomérico de silicona. La cantidad de furosemida con la que se carga el infusor viene determinada por la dosis diaria de furosemida a infundir y los días de tratamiento. En relación con la indicación de furosemida parenteral se consideró en este caso por insuficiente respuesta a terapia diurética oral combinada asociada a pulsos intravenosos de furosemida de forma ambulatoria en el servicio hospital de Día.

Desde el punto de vista sintomático, durante el tiempo que permaneció con esta modalidad terapéutica, más de cuatro meses, se consiguió un buen control de los síntomas congestivos, mejoró la función renal y la clase funcional, llegando a realizar pequeños paseos fuera de su domicilio, y no precisó ingresos hospitalarios.

En la experiencia del grupo, la tolerancia y adaptación por parte de los pacientes son buenas. Las primeras visitas de control se realizan habitualmente en las consultas del hospital hasta obtener un ajuste preciso de dosis y estabilidad metabólica en analítica, y posteriormente el seguimiento se continúa en casa por parte del equipo domiciliario de cuidados paliativos y médicos de atención primaria, evitando así el desplazamiento a consultas y el internamiento hospitalario, salvo complicaciones o requerimientos especiales del paciente.

\section{Conclusiones}

El tratamiento de la fase terminal de la insuficiencia cardiaca consiste prioritariamente en la asistencia para la toma de decisiones relacionada con las terapias y el lugar de cuidados, el soporte emocional y el control de síntomas para mantener el máximo posible la calidad de vida según valores y deseos del paciente. En cuanto al tratamiento de los síntomas congestivos, que suelen ser los más frecuentes, la administración parenteral continua de furosemida por vía subcutánea mediante infusores elastoméricos es una técnica sencilla que parece ser segura y efectiva, reduce la necesidad de internamiento hospitalario y facilita la permanencia de los pacientes en su domicilio. No obstante, aún son necesarios estudios que aporten más evidencia al respecto para recomendar de manera definitiva esta modalidad de tratamiento. Por el momento resulta indispensable individualizar la indicación atendiendo a deseos del paciente, soporte cuidador familiar y la necesaria colaboración con equipos de cuidados paliativos, atención primaria y hospitalización a domicilio, o ambos.

El interés de este caso reside en que se trata del único descrito con administración eficaz de furosemida subcutánea mediante sistema elastomérico superior a treinta días.

\section{Conflicto de intereses}

Ninguno.

\section{Bibliografía}

1. Mosterd A, Hoes AW. Clinical epidemiology of heart failure. Heart. 2007;93:1137-46.

2. Valente MA, Voors AA, Damman K, Van Veldhuisen DJ, Massie BM, O'Connor CM, et al. Diuretic response in acute heart failure: clinical characteristics and prognostic significance. Eur Heart J. 2014;35:1284-93.

3. Zatarain-Nicolás E, López-Díaz J, de la Fuente-Galán L, García Pardo H, Recio Platero A, San Román Calvar JA. Tratamiento de la insuficiencia cardiaca descompensada con furosemida subcutánea mediante bombas elastoméricas: experiencia inicial. Rev Esp Cardiol. 2013;66:1002-4.

4. Goenaga MA, Millet M, Sanchez E, Garde C, Carrera JA, Arzellus E. Subcutaneous furosemide. Ann Pharmacother. 2004;38: 1751.

5. Ponikowski P, Voors AA, Anker SD, Bueno H, Cleland JG, Coats AJ, et al. 2016 ESC Guidelines for the diagnosis and treatment of acute and chronic heart failure: The Task Force for the diagnosis and treatment of acute and chronic heart failure of the European Society of Cardiology (ESC). Developed with the special contribution of the Heart Failure Association (HFA) of the ESC. Eur J Heart Fail. 2016;18: 891-975.

6. Jaarsma T, Beattie JM, Ryder M, Rutten FH, McDonagh T, Mohacsi $P$, et al. Palliative care in heart failure: a position statement from the palliative care workshop of the Heart Failure Association of the European Society of Cardiology. Eur J Heart Fail. 2009;11:433-43.

7. Fendler TJ, Swetz KM, Allen LA. Team-based palliative and end of- life care for heart failure. Heart Fail Clin. 2015;11: 479-98.

8. Fonzo-Christe C, Vukasovic C, Wasilewski-Rasca AF, Bonnabry P. Subcutaneous administration of drugs in the elderly: survey of practice and systematic literature review. Palliat Med. 2005;19:208-19.

9. Goenaga MA, Millet M, Carrera JA. Garde C. Vía subcutánea: más fármacos. Med Paliat. 2000;1:28.

10. Zacharias H, Raw J, Nunn A, Parsons S, Johnson M. Is there a role for subcutaneous furosemide in the community and hospice management of end-stage heart failure? Palliat Med. 2011;25:658-63.

11. Beattie JM, Johnson MJ. Subcutaneous furosemide in advanced heart failure: has clinical practice run ahead of the evidence base? BMJ Support Palliat Care. 2012;2:5-6.

12. Francis GS, Alexy T. Furosemide reimagined: novel subcutaneous formulation for a 50-year-old loop diuretic agent for the treatment of acute decompensated heart failure. JACC Heart Fail. 2018;6:71-2. 